\title{
The Upregulation of a Novel Long Noncoding RNA AK097647 Promotes Enterovirus 71 Replication and Decreases IFN- $\boldsymbol{\lambda} 1$ Secretion
}

\author{
Min Chu ${ }^{a, b}$ Bingfei Zhou ${ }^{b}$ Huilin Tu ${ }^{b}$ Min Lic Li Huang $^{d}$ Yuan He $^{d}$ Luo Liu \\ Song Han ${ }^{a, b}$ Jun Yin ${ }^{a, b}$ Biwen Peng ${ }^{b}$ Xiaohua He ${ }^{b}$ Wanhong Liu ${ }^{a, b}$ \\ aShenzhen Institute of Wuhan University, Shenzhen, China; b Hubei Province Key Laboratory of Allergy and \\ Immunology, School of Basic Medical Sciences, Wuhan University, Wuhan, China; ${ }^{\circ}$ The Department of Clinical \\ Laboratory, Central Hospital of Huanggang, Huanggang, China; ${ }^{\mathrm{d}}$ The Medical Research for Structural Biology of Basic

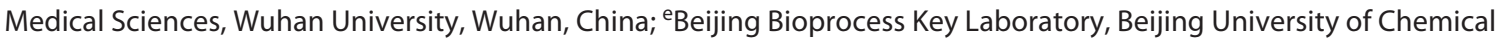 \\ Technology, Beijing, China
}

\section{Keywords}

Enterovirus 71 • Long noncoding RNA · IncRNA-AK097647 • IFN- $\lambda 1 \cdot$ Viral replication

\begin{abstract}
Background: Enterovirus 71 (EV71) infects millions of children every year in China and has become a challenge to public health. However, there is no effective treatment for EV71 infection. Long noncoding RNAs (IncRNAs) have been found to play various roles in virus replication and infection. Objective: We aimed to explore the role of a novel long noncoding RNA AK097647 (IncRNA-AK097647) during EV71 infection. Methods: To assess the role of IncRNA-AK097647 during EV71 infection, siRNAs were used to silence IncRNA-K097647 expression. RT-qPCR assay and Western blotting were applied to measure the mRNA and protein levels of EV71 VP1 and the phosphorylation of NF-KB. ELISA was used to detect the level of IFN- $\lambda 1$ expression. Results: The novel IncRNAAK097647 was upregulated in human rhabdomyosarcoma cells and the blood of hand, foot, and mouth disease patients infected with EV71, as demonstrated by RT-qPCR. Interestingly, RNAi-mediated knockdown of IncRNA-AK097647 dramatically increased the level of IFN- $\lambda 1$ expression, resulting
\end{abstract}

in the suppression of EV71 replication. In contrast, overexpression of IncRNA-AK097647 decreased the level of IFN- $\lambda 1$ expression and resulted in increased EV71 replication. In addition, we found that IncRNA-AK097647 could inhibit the phosphorylation of NF-KB. Conclusion: These results suggest a novel mechanism by which EV71 evades the IFN-mediated host antiviral response by increasing IncRNAAK097647 expression.

(c) 2021 The Author(s).

Published by S. Karger AG, Basel

\section{Introduction}

Enterovirus 71 (EV71), a single-stranded positivesense RNA virus, belongs to the Enterovirus genus of the Picornaviridae family. It is a major viral pathogen of severe hand, foot, and mouth disease (HFMD) and may cause neurotropic manifestations such as aseptic meningitis, encephalitis, and neurogenic pulmonary edema in children $<5$ years, even causing death in severe cases [1, 2]. EV71 outbreaks are frequently reported in Southeast Asia and China [3, 4]. Approximately 2 million children are diagnosed with HFMD in China each year, and no specific antiviral therapy for EV71 infection is currently
C 2021 The Author(s).

Published by S. Karger AG, Basel

This is an Open Access article licensed under the Creative Commons Attribution-NonCommercial-4.0 International License (CC BY-NC) (http://www.karger.com/Services/OpenAccessLicense), applicable to the online version of the article only. Usage and distribution for commercial purposes requires written permission.
Correspondence to:

Wanhong Liu, liuwanhong@whu.edu.cn 
available. Thus, EV71 infection is a severe threat to public health in China and even the world.

Long noncoding RNAs (lncRNAs) are a large number of nonprotein-coding transcripts that are $>200$ bases in length $[5,6]$. lncRNAs are involved in diverse biological aspects, such as mediating the regulation of cell signaling in cancer, playing an essential role in metastasis and neurodegenerative diseases, and participating in the host cell response toward bacterial infections [7-9]. Several lncRNAs have been described as playing relevant roles in viral infections and in the antiviral response. It has been reported that lncRNA-ACOD1 affects the cellular metabolism to promote viral infection and that NEAT1 serves as a positive feedback mechanism for RIG-I signaling to exert its anti-hantaviral effects $[10,11]$. To identify functional lncRNAs correlating with EV71, 3 microarray studies were performed on comprehensive lncRNA profiling analyses in EV71-infected human rhabdomyosarcoma (RD) cells and severe EV71-infected HFMD patients [12-14]. However, the functions and detailed mechanisms of lncRNAs during EV71 infection are still unclear.

In this study, we focused on the characterization of a novel lncRNA-AK097647, a human lncRNA located on chromosome 5q33.3. We showed that lncRNA-AK097647 expression gave rise to the reduction in the mRNA level of IFN- $\lambda 1$, the inhibition of IFN- $\lambda 1$ secretion, and a decrease in the phosphorylation of NF- $\kappa \mathrm{B}$ in human RD cells, thus resulting in a remarkable increase in the replication of EV71. These data emphasize the positive role of lncRNA-AK097647 in contributing to EV71 replication, suggesting that lncRNA-AK097647 might be a target for EV71 therapy.

\section{Material and Methods}

\section{Clinical Blood Samples}

Blood samples were obtained from 6 patients with EV71 infection and 10 healthy individuals. Total RNA was extracted and then used for RT-qPCR analysis.

a. Ethical approval number and ethical committee

The ethical approval number is 18005 . The study was conducted according to the principles of the Declaration of Helsinki and approved by the Institutional Review Board of the College of Basic Medical Sciences, Wuhan University, in accordance with the guidelines for the protection of human subjects. Written informed consent was obtained from each participant.

b. RNA extraction

Total RNA was extracted with TRIzol Reagent (Invitrogen, Carlsbad, CA, USA). First, $0.75 \mathrm{~mL}$ TRIzol reagent was added to 0.25 $\mathrm{mL}$ blood sample and then incubated at room temperature for $5 \mathrm{~min}$. And $0.2 \mathrm{~mL}$ chloroform/1 mL TRIzol was added, and tubes were shaken vigorously by hand for $15 \mathrm{~s}$ and then incubated at room temperature for $3 \mathrm{~min}$. It was then centrifuged $15 \mathrm{~min}$ at $4^{\circ} \mathrm{C}$ at $12,000 \mathrm{~g}$. RNA is on top. The RNA aqueous phase was transferred to a fresh tube, and $0.5 \mathrm{~mL}$ isopropanol/ $\mathrm{mL}$ TRIzol was added. Then, it was incubated $10 \mathrm{~min}$ at room temperature and centrifuge $10 \mathrm{~min}$ at $4^{\circ} \mathrm{C}$ at $12,000 \mathrm{~g}$, and the supernatant was removed and disposed. RNA pellet was washed once with $75 \%$ ethanol $1 \mathrm{~mL} / \mathrm{mL}$ TRIzol and vortexed and centrifuged $5 \mathrm{~min}$ at $4^{\circ} \mathrm{C}$ at $12,000 \mathrm{~g}$. RNA pellet was saved. Finally, RNA was dissolved in $0.03 \mathrm{~mL}$ RNase-free water.

c. RT-qPCR primers and conditions

RT-qPCR was performed with SYBR Green Real-Time PCR Master Mix (Toyobo, Osaka, Japan) according to the manufacturer's instructions. GAPDH was used as an internal control, and the RT-qPCR primers used were as follows: VP1 (forward: CTCTAAAGGGCACCACCAATC; reverse: AAGTGAACTCTGCGTCAAAGC); initial denaturation at $95^{\circ} \mathrm{C}$ for $30 \mathrm{~s}$, then $95^{\circ} \mathrm{C}$ for $5 \mathrm{~s}, 56^{\circ} \mathrm{C}$ for $15 \mathrm{~s}$, and $72^{\circ} \mathrm{C}$ for $10 \mathrm{~s}$ total 40 cycles.

\section{EV71 Infection}

RD cells were infected with EV71 (BrCr strain, GenBank Accession No. U22521) at a multiplicity of infection of 10 and incubated for $1 \mathrm{~h}$ at $37^{\circ} \mathrm{C}$. Then, the inoculum was removed, and incubation was continued in fresh culture medium supplemented with $1 \%$ heat-inactivated $\mathrm{FBS}$ at $37^{\circ} \mathrm{C}$ and $5 \% \mathrm{CO}_{2}$. The infected cells and culture supernatants were collected together at day 7 postinfection and clarified by centrifugation $(2,000 \mathrm{~g})$. Finally, virus titers were determined by plaque assays.

\section{Plasmid and siRNA}

The lncRNA-AK097647 recombinant plasmid was subcloned into the pcDNA3.1 (-) vector (Promega, Madison, WI, USA). The primers used for plasmid construction were as follows: (forward: AATCTAGAGTCCTCGAGGAGTCTCTCG; reverse: GGGGATCCAGAGTGGTAACTGTTTAATG). Si-AK097647 and the negative control were purchased from GenePharma (Suzhou, China).

\section{RNA Extraction and RT- $q P C R$}

Total RNA was extracted with TRIzol reagent (Invitrogen, Carlsbad, CA, USA) and reverse transcribed using a RevertAid First Strand cDNA Synthesis Kit (Thermo Scientific, Waltham, MA, USA). Nuclear and cytoplasmic extractions were isolated using the nuclear and cytoplasmic extraction kit from Thermo Scientific (following the manufacturer's specification). RT-qPCR was performed with SYBR Green Real-Time PCR Master Mix (Toyobo, Osaka, Japan) according to the manufacturer's instructions. GAPDH was used as an internal control, and the RT-qPCR primers used were as follows: VP1 (forward: CTCTAAAGGGCACCACCAATC; reverse: AAGTGAACTCTGCGTCAAAGC); AK097647 (forward: TGTGCGGTTCTTGTGCTAGT; reverse: GACCCAAAGGAGTGGATGAA); GAPDH (forward: CAAGAGCACAAGAGGAAGAGAG; reverse: GGTTGAGCACAGGGTACTTTAT); IFN $\beta$ (forward: GCTCTCCTGTTGTGCTTCTC; reverse: AGTCTCATTCCAGCCAGTGC); IFN- $\lambda 1$ (forward: GAGACCTCAAATATGTGG; reverse: GCATAAATAAGGTGTGGG); IFN- $\lambda 2$ (forward: ACGCGAGACCTGAATTGTGT; reverse: AGCGACTGGGTGGCAATAAA); and U1 (forward: TCCCAGGGCGAGGCTTATCCATT; reverse: GAACGCAGTCCCCCACTACCACAAAT).
Chu/Zhou/Tu/Li/Huang/He/Liu/Han/ Yin/Peng/He/Liu 
Fig. 1. $\ln \mathrm{RNA}-\mathrm{AK} 097647$ is increased during EV71 infection. a RT-qPCR analysis of lncRNA-AK097647 expression in human RD cells infected with EV71 (MOI = 10). b RT-qPCR analysis of lncRNAAK097647 expression in EV71 patients' blood samples $(n=6)$ and healthy blood samples $(n=10)$. c Analysis obtained from the Coding Potential Calculator based on evolutionary conservation and ORF attributes. d RT-qPCR detection of lncRNAAK097647 in the cytoplasmic and nuclear fractions of RD cells. GAPDH and U1 served as cytoplasmic and nuclear localization controls, respectively. e RNA FISH assay of lncRNA-AK097647 localization in RD cells. Scale bar, $5 \mu \mathrm{m}$. The $18 \mathrm{~S}$ probe was used as a cytoplasmic positive control, and the U6 probe was used as a nuclear positive control. EV71; enterovirus 71; lncRNA, long noncoding RNA; RD, rhabdomyosarcoma; MOI, multiplicity of infection.

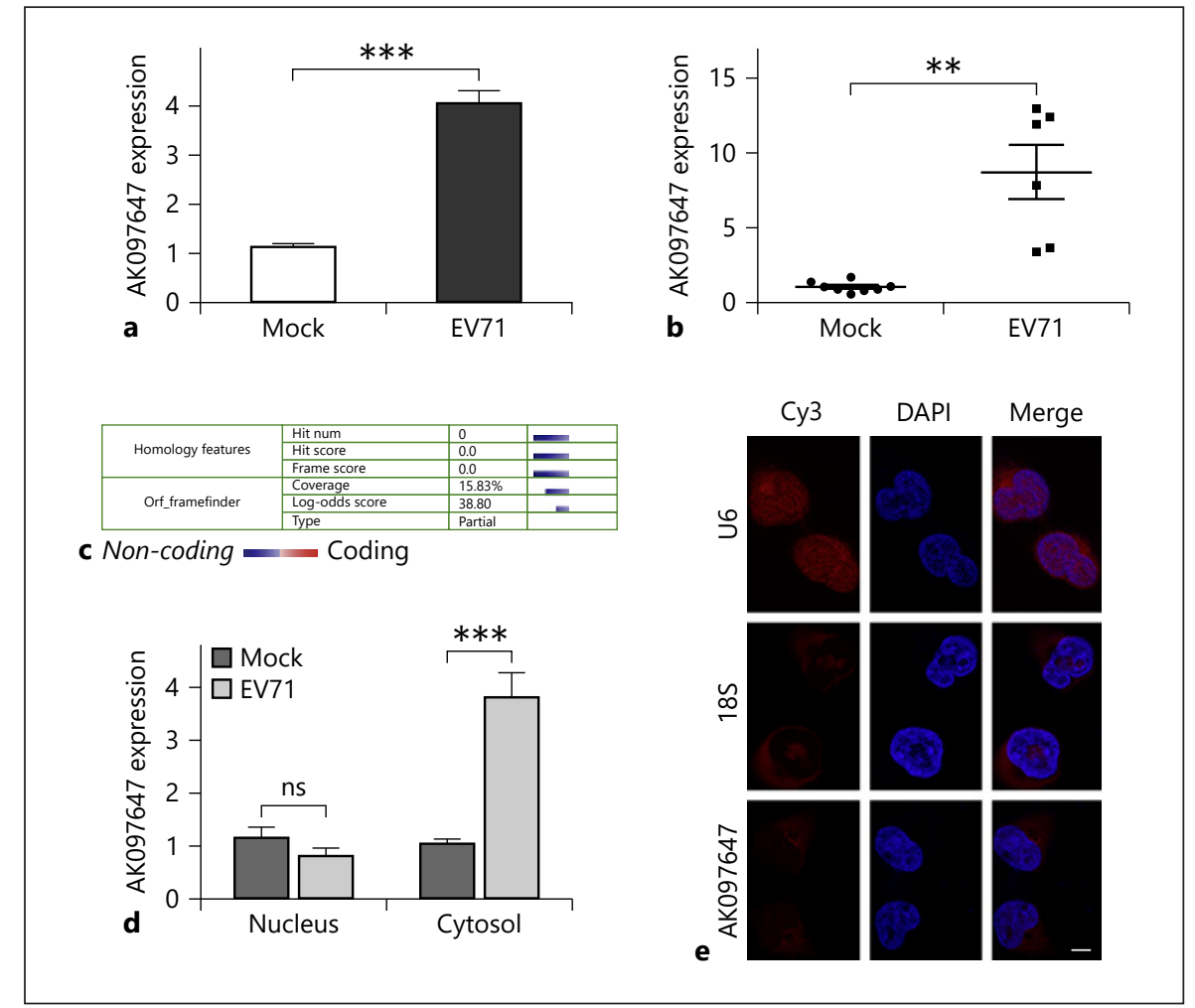

Plaque Assay

Vero cell monolayers were seeded at a concentration of $1 \times 10^{5}$ cells per $\mathrm{mL}$ in DMEM supplemented with $10 \%$ heat-inactivated FBS. The cells were incubated with $200 \mu \mathrm{L}$ of a serial 10 -fold-diluted viral suspension for $1 \mathrm{~h}$, and the viral solution was aspirated. Subsequently, $500 \mu \mathrm{L}$ of DMEM supplemented with $1 \%$ heat-inactivated FBS and antibiotics plus $1 \%$ carboxymethyl cellulose was added to the wells. Then, the plates were incubated at $37^{\circ} \mathrm{C}$ and $5 \%$ $\mathrm{CO}_{2}$ for $3 \mathrm{~d}$, and the cells were fixed with methanol at $4^{\circ} \mathrm{C}$ for $1 \mathrm{~h}$ and subsequently stained with crystal violet for $10 \mathrm{~min}$.

\section{Confocal Microscopy}

$\mathrm{RD}$ cells were plated in confocal dishes (NEST Biological Technology, Shanghai, China) at a density of $1 \times 10^{6}$ cells per dish, and after $20 \mathrm{~h}$, the cells were fixed with $4 \%$ paraformaldehyde for 10 min, and cells were washed 3 times with PBS. Then, cells were treated with $1 \%$ Triton for 5 min. After washing 3 times with PBS, the cells were incubated with lncRNA FISH Probe Mix (RIBOBIO, Guangzhou, China) at $37^{\circ} \mathrm{C}$ overnight. Then, nuclei were stained with DAPI in $1 \times$ PBS for 5 min at RT. The probe labeled with $\mathrm{Cy} 3$ was excited with a 555-nm diode laser and detected using the 570$\mathrm{nm}$ long-pass filter. Confocal laser scanning was performed using a Leica-LCS-SP8-STED.

\section{Western Blotting and Cytokine Analysis}

RD cells were lysed with RIPA buffer (Biosharp, China). Then, the cell lysates were collected and loading buffer was added to it. The protein samples were separated in $12 \%$ polyacrylamide gels and then transferred to PVDF membranes (Millipore, Hertfordshire, UK). The membranes were blocked with $5 \%$ bovine serum albumin in Tris-buffered saline containing $0.1 \%$ Tween 20 at room temperature for $2 \mathrm{~h}$ and then incubated overnight at $4^{\circ} \mathrm{C}$ with the following primary antibodies (except for incubations with the mouse polyclonal antibody to EV71 for $48 \mathrm{~h}$ ): mouse polyclonal antibody against EV71 (1 $\mu \mathrm{g} / \mathrm{mL}$; ab169442, Abcam, Cambridge, MA, USA), rabbit anti-phospho-NF- $\kappa B$ p65 (Ser536) $(1 \mu \mathrm{g} / \mathrm{mL}$; \#3033, Cell Signaling Technology), rabbit anti-NF- $\kappa$ B p65 (1 $\mu \mathrm{g} /$ $\mathrm{mL}$ \#8242, Cell Signaling Technology), and anti-GAPDH mouse monoclonal antibody (diluted 1:5,000; PMK043C, PMK, Wuhan, China). Protein band signals were detected by enhanced chemiluminescence (Thermo Scientific, Waltham, MA, USA). Band densities were measured by densitometry and quantified using ImageJ software (version $1.46 \mathrm{r}$, Bethesda, MD, USA). The IFN- $\lambda 1$ protein level in the cell supernatants was detected with an ELISA kit (BioSwamp, China).

\section{Statistical Analysis}

All experiments were performed in triplicate. The data are expressed as mean \pm standard derivation and were analyzed by Student's $t$ test. Significant differences were indicated by a $p<0.05$.

\section{Results}

\section{IncRNA-AK097647 Expression Was Increased during EV71 Infection}

The expression level of lncRNA-AK097647 was detected by RT-qPCR. We found that lncRNA-AK097647 
Fig. 2. Overexpression of lncRNAAK097647 promotes EV71 infection. a Virus titer regulated by lncRNA-AK097647 using plaque assay for the EV71 BrCr strain in a 24 -well plate. b EV71 VP1 mRNA expression levels were tested by RT-qPCR. c EV71 VP1 protein expression levels were tested by Western blotting. d Cytotoxicity of pcDNA3.1 (-) and pcDNA3.1 (-)-lncRNA-AK097647 on RD cells was tested by the CCK8 kit. EV71; enterovirus 71; lncRNA, long noncoding RNA; RD, rhabdomyosarcoma.

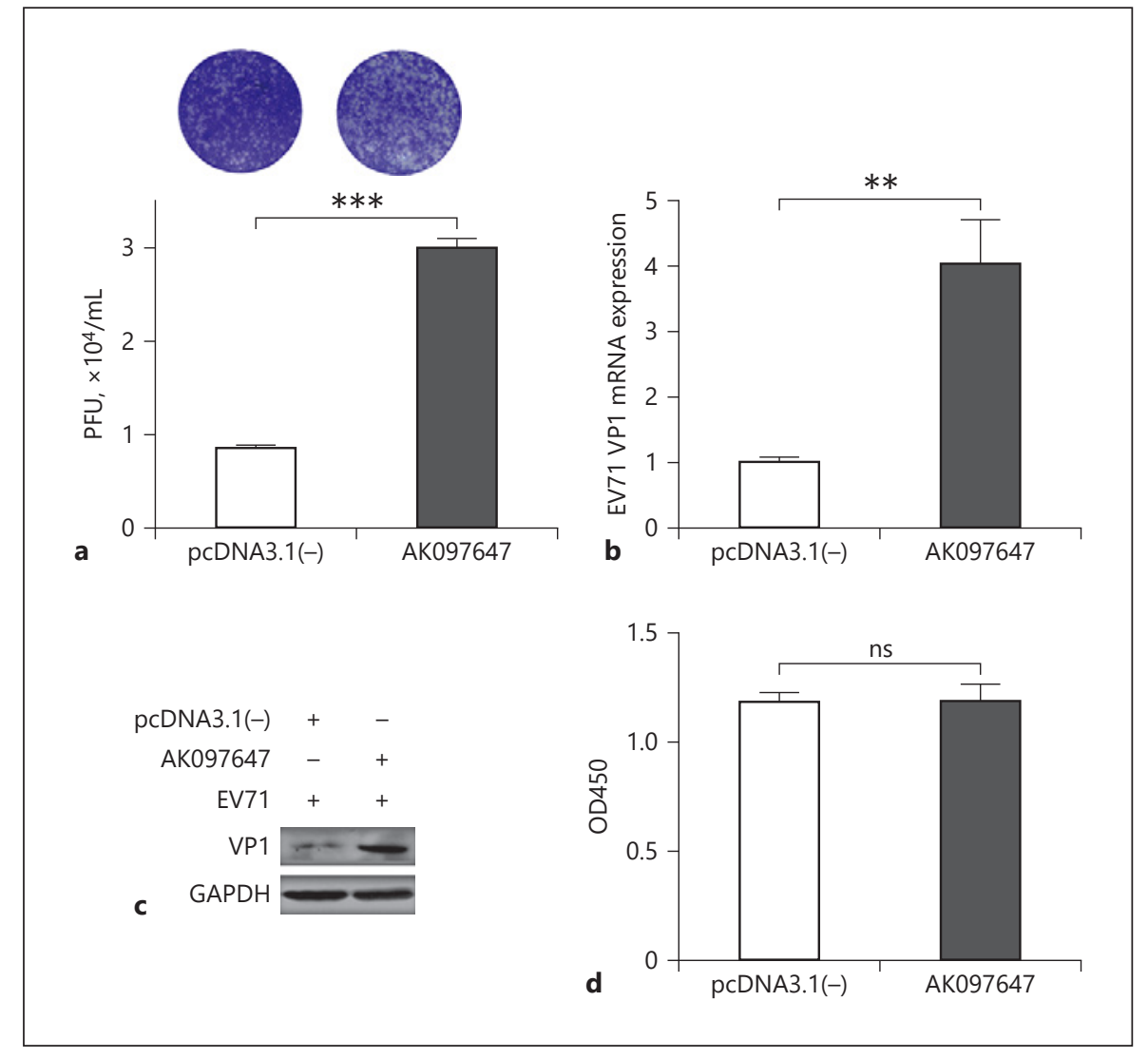

was consistently upregulated 3.6-fold in EV71-infected $\mathrm{RD}$ cells compared with the mock group (Fig. 1a). The expression level of IncRNA-AK097647 was increased 8.3fold in EV71-infected patients compared with the healthy control group (Fig. 1b). Based on the information in GenBank, lncRNA-AK097647 is a 1,668-nucleotide intronless transcript. No protein-coding potential was found in AK097647 by analysis using CPAT (score was 0.0079) (Fig. 1c). Further detection of lncRNA-AK097647 in the cytoplasmic and nuclear fractions of RD cells showed that lncRNA-AK097647 was increased 3.7-fold in the cytoplasm of EV71-infected cells compared with mock-infected cells, while there were no differences in the nuclear level of lncRNA-AK097647 between EV71-infected cells and mock-infected cells (Fig. 1d). Moreover, confocal microscopy results showed that lncRNA-AK097647 was located primarily in the cytoplasm, rather than in the nucleus (Fig. 1e).

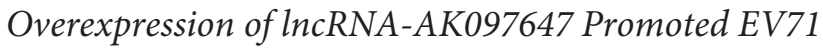
Infection

To determine whether lncRNA-AK097647 expression might reciprocally influence EV71 replication, lncRNA-
AK097647 was overexpressed in RD cells followed by EV71 infection. The viral titer in the culture supernatants of lncRNA-AK097647-overexpressing cells was on the order of $3 \times 10^{4} \mathrm{PFU} / \mathrm{mL}$, which was 3.4 -fold higher than that in the control cells $\left(0.88 \times 10^{4} \mathrm{PFU} / \mathrm{mL}\right.$; Fig. 2a). The EV71 genomic RNA was 4.0-fold higher in the lncRNAAK097647-overexpressing groups than in the negative control groups (Fig. 2b). The EV71 VP1 protein expression level was increased in the lncRNA-AK097647-overexpressing groups compared with the negative control (Fig. 2c). In addition, the cell viability in the IncRNAAK097647 group and the vector control group showed no differences using the CCK8 assay (Fig. 2d).

\section{Knockdown of IncRNA-AK097647 Inhibited EV71 Infection}

To further confirm the function of IncRNA-AK097647 in EV71 replication, RNA interference to knock down lncRNA-AK097647 was used. The level of endogenous lncRNA-AK097647 was decreased by $78 \%$ by transfection with siAK097647\#1 compared with the negative control group, but the siAK097647\#2 transfection groups showed no difference compared to the negative control groups 
Fig. 3. Knockdown of lncRNA-AK097647 inhibits EV71 infection. a RD cells were transfected with $20 \mathrm{pmol}$ of $\mathrm{NC}$, siAK097647\#1, or siAK097647\#2, and relative lncRNA-AK097647 expression was determined by RT-qPCR. b RD cells were transfected with 20 pmol of NC, siAK097647\#1, or siAK097647\#2, and subsequently infected with EV71 (MOI = 10). EV71 VP1 mRNA expression levels were tested by RT-qPCR. c Virus titer regulated by siAK097647\#1 using plaque assay for the EV71 BrCr strain in a 24-well plate. RD cells were transfected with $20 \mathrm{nM}$ NC or siAK097647\#1 and subsequently infected with EV71 (MOI = 10). Plaques were stained by crystal violet. d EV71 VP1 protein expression levels were tested by Western blotting 48 hpi. e Cytotoxicity of NC or siAK097647\#1 on RD cells determined by the CCK8 kit. EV71; enterovirus 71; lncRNA, long noncoding RNA; RD, rhabdomyosarcoma; MOI, multiplicity of infection; hpi, hour post-infection.
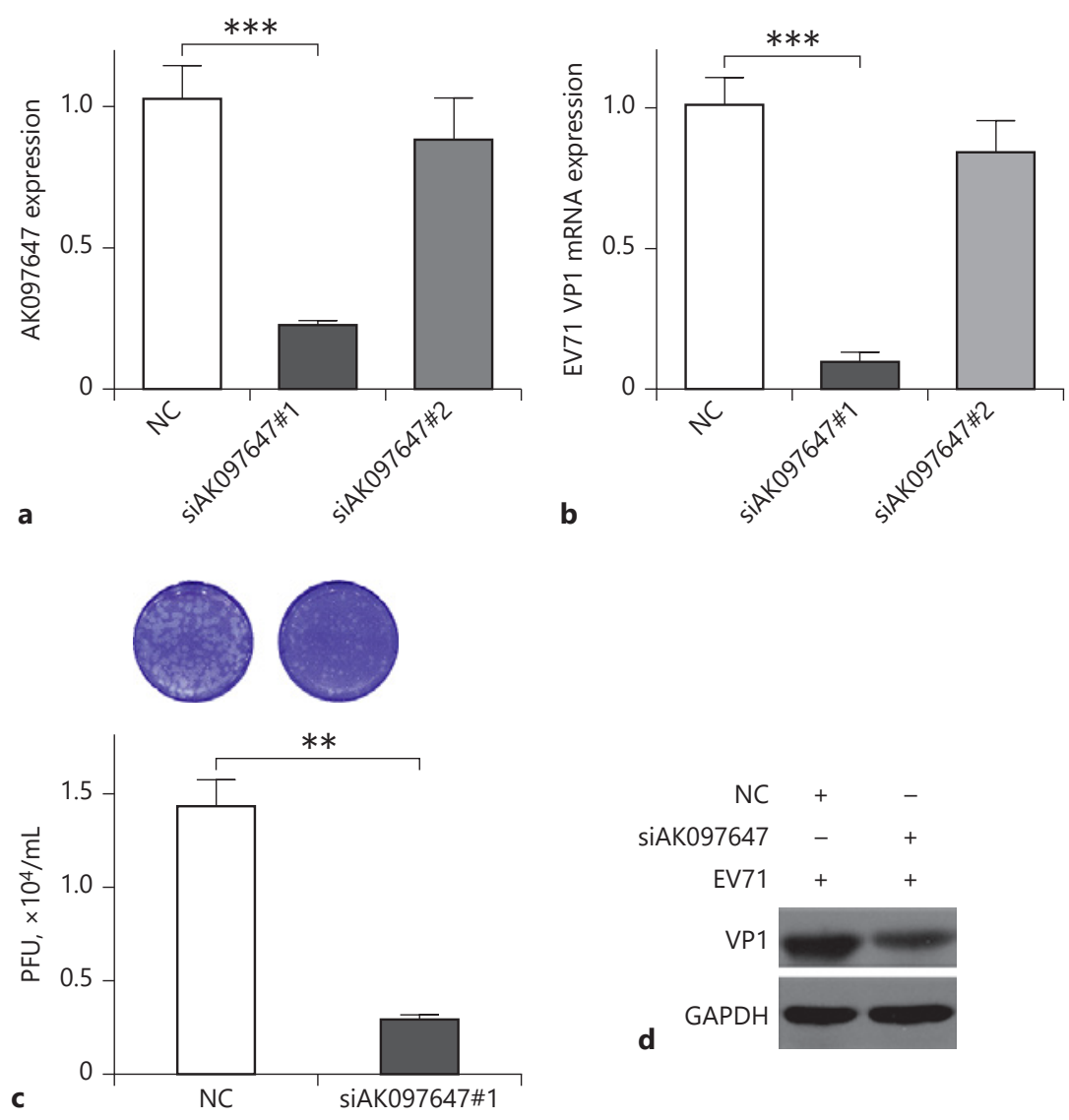

C

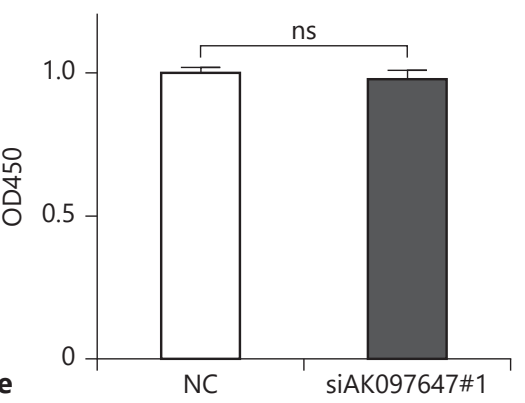

(Fig. 3a). EV71 genomic mRNA expression was 90\% lower in the siAK097647\#1 knockdown group than in the negative control group, while the siAK097647\#2 transfection group showed no difference from the negative control group (Fig. 3b). Therefore, we used siAK097647\#1 for the next experiment. The viral titer in the culture supernatants of siAK097647\#1-transfected cells was $0.29 \times$ $10^{4} \mathrm{PFU} / \mathrm{mL}$, which was 4.9 -fold lower than that in the control $\left(1.43 \times 10^{4} \mathrm{PFU} / \mathrm{mL}\right.$; Fig. $\left.3 \mathrm{c}\right)$. The EV71 VP1 protein expression level was also decreased in the siAK097647\# 1 group compared with the negative control group (Fig. 3d). In addition, the cell viability in the siAK097647\#1 group and the negative control group showed no differences using the CCK8 assay (Fig. 3e).

\section{IncRNA-AK097647 Blocked Interferon- $\lambda 1$ Secretion during EV71 Infection}

To determine whether the function of lncRNAAK097647 was dependent on interferons, RD cells were transfected with pcDNA3.1 (-) vector or pcDNA3.1 (-)-IncRNA-AK097647, followed by EV71 infection for $24 \mathrm{~h}$, and the mRNA expression levels of the antiviral cy- 

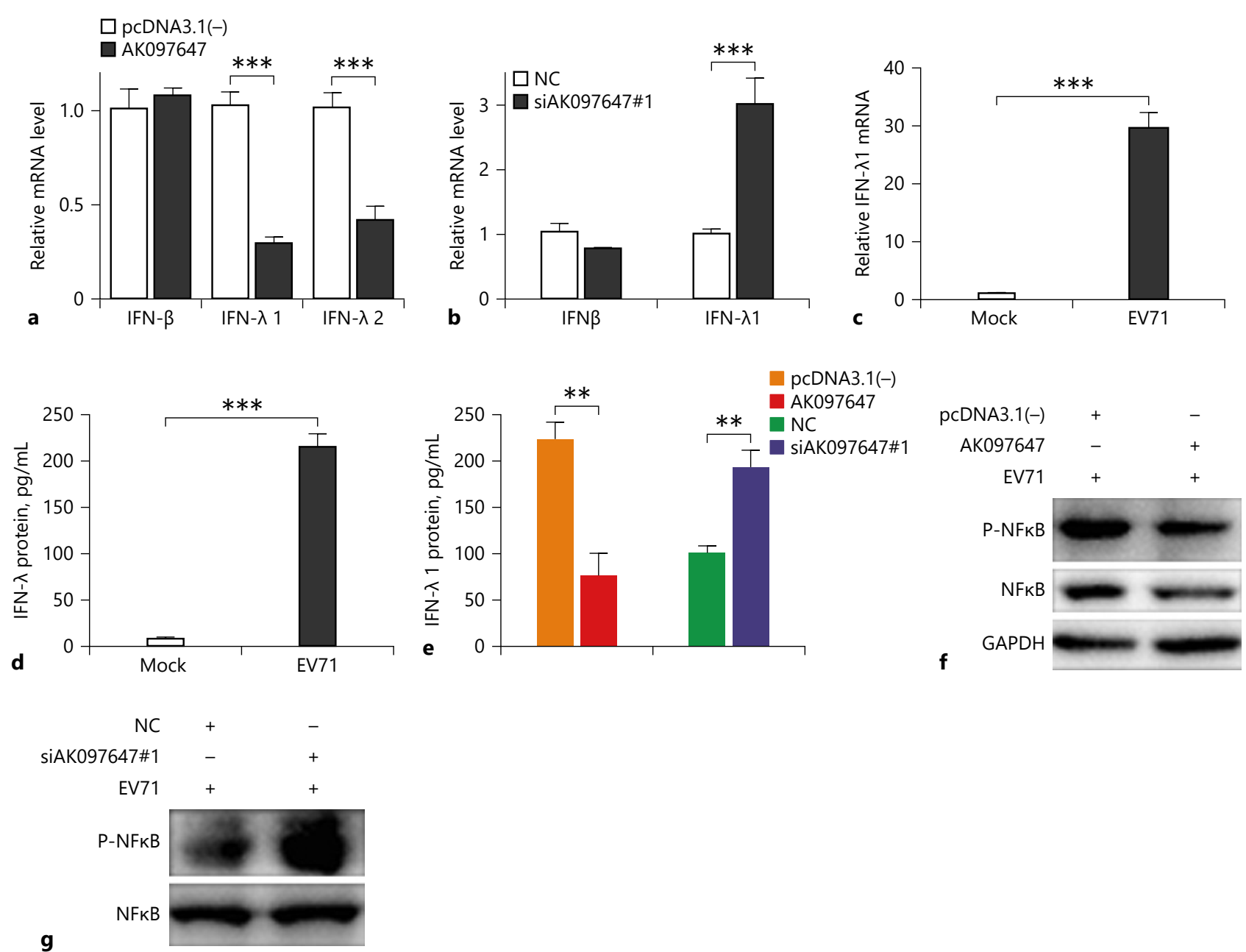

Fig. 4. $\ln c \mathrm{RNA}-\mathrm{AK} 097647$ blocks interferon- $\lambda 1$ secretion in $\mathrm{RD}$ cells during EV71 infection. a RD cells were transfected with $1 \mu \mathrm{g} /$ mL pcDNA3.1 (-) or pcDNA3.1 (-)-lncRNA-AK097647 for $24 \mathrm{~h}$. Then, the cells were infected with EV71 for $24 \mathrm{~h}$, and the cell lysates were used to determine the relative mRNA levels of IFN, IFN- $\lambda 1$, and IFN- $\lambda 2$ by RT-PCR. $\mathbf{b}$ RD cells were transfected with 20 pmol of NC or siAK097647\#1 for $36 \mathrm{~h}$ and then infected with EV71 $(\mathrm{MOI}=10)$ for $24 \mathrm{~h}$. The cell lysates were used to determine the relative mRNA levels of IFN $\beta$ and IFN- $\lambda 1$ by RT-qPCR. c RT-qPCR analysis of IFN- $\lambda 1$ expression in human RD cells infected with EV71 $(\mathrm{MOI}=10)$. These experiments were performed in triplicate.

tokines IFN- $\beta$, IFN- $\lambda 1$, and IFN- $\lambda 2$ were assessed. These results showed that overexpression of lncRNA-AK097647 reduced the IFN- $\lambda 1$ mRNA level by $71 \%$ and the IFN- $\lambda 2$ mRNA level by $58 \%$, but IFN- $\beta$ mRNA expression had no differences (Fig. 4a). Next, siAK097647\#1 was transfected into RD cells for $36 \mathrm{~h}$, and then, these cells were infected with EV71 for $24 \mathrm{~h}$. Conversely, we found that the IFN- $\lambda 1$ d The cell culture supernatants of (c) were used for IFN- $\lambda 1$ detection by ELISA. $\mathbf{e}$ The cell culture supernatants of both (a) and (b) were used for IFN- $\lambda 1$ detection by ELISA. $\mathbf{f}$ Phosphorylation of NF- $\kappa B$ p 65 Ser 536 and NF- $\kappa B$ protein expression levels were tested by Western blotting $24 \mathrm{hpi}$. $\mathrm{g}$ RD cells were transfected with 20 pmol of NC or siAK097647\#1 for $36 \mathrm{~h}$ and then infected with EV71 $(\mathrm{MOI}=10)$ for $24 \mathrm{~h}$. Phosphorylation of NF-kB p65 Ser536 and NF- $\mathrm{kB}$ protein expression levels were tested by Western blotting. EV71, enterovirus 71; lncRNA, long noncoding RNA; RD, rhabdomyosarcoma; MOI, multiplicity of infection; hpi, hour post-infection.
mRNA level in the siAK097647\#1-transfected group was 3 -fold higher than that in the negative control group, while IFN- $\beta$ mRNA expression showed no significant change (Fig. $4 \mathrm{~b}$ ). To test whether RD cells are responding to IFN- $\lambda 1$ or not, we used RT-PCR and ELISA methods and found $\mathrm{RD}$ cells are responding to IFN- $\lambda 1$ during EV71 infection (Fig. 4c, d). We also found that the mean 
level of IFN- $\lambda 1$ protein in the lncRNA-AK097647 overexpression group was $76.45 \mathrm{pg} / \mathrm{mL}$ which was 2.93 -fold lower than that of the vector group $(224.10 \mathrm{pg} / \mathrm{mL})$ at 24 $h$ post-infection. The mean level of IFN- $\lambda 1$ protein in the siAK097647\#1 group was $192.63 \mathrm{pg} / \mathrm{mL}$ which was 1.92fold higher than that of the negative control group (100.36 $\mathrm{pg} / \mathrm{mL}$ ), at $24 \mathrm{~h}$ post-infection (Fig. 4e). Our exploration also showed that the phosphorylation of the NF- $\kappa B-p 65$ protein was markedly decreased in lncRNA-AK097647overexpressing RD cells during EV71 infection (Fig. 4f). The phosphorylation of NF- $\kappa \mathrm{B}-\mathrm{p} 65$ protein was significantly increased in lncRNA-AK097647-knockdown RD cells during EV71 infection (Fig. 4g).

\section{Discussion}

The broad spectrum of activities and versatile regulatory mechanisms of lncRNAs indicate that lncRNAs play key roles in host innate immunity during viral infection. For example, IncRNA NRAV significantly promotes Influenza A virus replication and virulence by negatively regulating the initial transcription of multiple critical interferon-stimulated genes, including IFITM3 and MxA [15]. IncRNA NRON contributes to HIV-1 latency by directly linking Tat to the ubiquitin/proteasome components CUL4B and PSMD11, thus facilitating Tat degradation [16]. Herpes simplex virus-1 infection increases the expression of lncRNA NEAT1 in a STAT3-dependent manner, and HSV-1 can use NEAT1 and paraspeckles, subnuclear structures, to facilitate HSV-1 gene expression and enhance viral replication [10]. Here, we showed that lncRNA-AK097647 contributed to EV71 replication through the degradation of IFN $-\lambda 1$. Some human and nonhuman short noncoding RNA with direct antiviral activity, for example, a short hairpin RNA, was found to target the noncoding region of the Influenza A virus RNA gene-encoding nucleoprotein to inhibit Influenza A virus infection [17]. Ril32, an intracellularly expressed noncoding small RNAs, was found to have the possibility to induce IFN- $\beta$ expression to inhibit influenza virus replication [18]. These studies indicated that noncoding RNAs play key roles in host antiviral immunity. In the study, we showed that a novel lncRNA-AK097647 contributed to EV71 replication through the inhibition of IFN- $\lambda 1$ expression.

Although Yin et al. [12] reported that 2,990 lncRNAs are upregulated and 1,876 $\mathrm{lncRNAs}$ are downregulated in EV71-infected RD cells compared with mock groups by using $\operatorname{lncRNA}$ microarray analyses, there is still no report

lncRNA-AK097647 Regulating EV71

Replication on how lncRNAs play the functional role during EV71 infection [14]. In our study, we found that IncRNAAK097647 expression was increased during EV71 infection, and this might be a positive feedback mechanism which lncRNA-AK097647 could contribute to EV71 replication in human $\mathrm{RD}$ cells. In recent studies, lncRNAs have been revealed to cis-regulate the transcription and translation of chromosomally adjacent genes. It was reported that lncRNA CASC15 regulates the expression of its chromosomally adjacent gene SOX4 [19]. We found that the adjacent gene of lncRNA-AK097647 is SOX30 using the online website UCSC Genome Browser (http:// genome.ucsc.edu/). Therefore, we examined whether lncRNA-AK097647 functions by affecting the nearby gene SOX30 (online suppl. Fig. S1A; for all online suppl. material, see www.karger.com/doi/10.1159/000515903). We found that both the overexpression and knockdown of lncRNA-AK097647 did not affect SOX30 protein expression (online suppl. Fig. S1B, C). Autophagy has been reported to participate in viral clearance [20-22]. Recent studies have shown that IncRNA MALAT1 modulates autophagy in different ways and is a potential regulator of autophagy [21-23]. Therefore, we examined whether lncRNA-AK097647 functions through autophagy. We tested the effect of IncRNA-AK097647 using Western blotting and found that both overexpression and knockdown of lncRNA-AK097647 had no influence on host cell autophagy (online suppl. Fig. S1D, E).

Type I IFNs (IFN- $\alpha,-\beta,-\kappa,-\varepsilon$, and $-\omega$ ) and type III IFNs (IFN- $\lambda 1$, IFN- $\lambda 2$, IFN- $\lambda 3$, and IFN- $\lambda 4$ ) provide a first line of defense in antiviral innate immune responses. Type I and type III IFNs show significant overlapping antiviral responses in the signaling cascade and relative interferon-stimulated genes. The main differences between the 2 kinds of IFNs are the IFN receptors and the cell types in which they are expressed. The type I IFN receptors are interferon receptor 1 (IFNAR1) and IFNAR2 and are broadly expressed on the majority of cell types, while the type III IFN receptors consisting of IFNLR1 and IL10R $\beta$ are predominantly expressed in epithelial cells and intestinal epithelial cells and are abundantly expressed in the liver and lung tissues [24]. Although many viruses such as hepatitis $C$ virus, hepatitis E virus, and HIV are restricted by IFNs at the initial stage of host cell infection, other viruses such as filoviruses, dengue virus, influenza virus [25], West Nile virus [26], and EV71 have evolved mechanisms to evade IFN attacks through the reduction in IFN synthesis, decrease in IFN secretion, deletion of IFN receptors, or blockage of IFN signaling. RSV-induced production of prostaglandin D2 was found to suppress IFN- $\lambda$ 
production [27]. Zika virus nonstructural protein NS5 was found to inhibit the RIG-I pathway and IFN- $\lambda 1$ promoter activation by targeting IKKe [28]. Npro of classical Swine fever virus suppressed IFN- $\lambda$ production by inhibiting IRF1 expression and its nuclear translocation [29]. In this study, we investigated whether IncRNA-AK097647 can play a role in host innate immunity during EV71 infection. We found that the overexpression of lncRNAAK097647 led to a reduction in the mRNA level of IFN- $\lambda$ but had no effect on IFN- $\beta$. And the IFN- $\lambda 1$ level in the siAK097647\#1-transfected group was higher than that in the negative control group, while IFN- $\beta$ expression showed no significant change. The possible mechanisms of lncRNA-AK097647 on regulating virus replication is that lncRNA-AK097647 may target IKKe or inhibit IRF1 expression and its nuclear translocation to suppressed IFN- $\lambda$ promoter activation and production, and further work is needed to explore the possible mechanisms.

The mechanism by which EV71 antagonizes interferon signaling to contribute to replication has been controversial. One study found that EV71 blocks JAK/STAT signaling by inducing karyopherin-al degradation to suppress interferon responses [30]. Another study found that EV71 inhibits the cellular type I IFN antiviral pathway by downregulating JAK1, but the expression of IFNAR1 was not significantly altered in EV71-infected cells [31]. Others found that EV71 antagonized type I IFN signaling by reducing the level of IFNAR1 [32]. MicroRNA-548 was found to downregulate the host antiviral response via direct targeting of IFN- $\lambda 1$ to promote EV71 replication [33]. We then found that knockdown of IncRNAAK097647 increased the phosphorylation of NF- $\kappa B$ protein levels. The activation of NF- $\mathrm{BB}$ protein phosphorylation mainly results in the translocation of NF- $\kappa B$ to the nucleus where it can bind NF- $\kappa B$-responsive DNA elements, recruit the transcription coregulators, and activate IFN- $\lambda 1$ expression. Although we found the role of $\ln$ cRNA-AK097647 in host-virus interactions, future investigations into how lncRNA-AK097647 interacts with IFN- $\lambda$ s should be performed, and the upstream mechanisms of transcriptional regulation of IncRNA-AK097647 should be elucidated.

\section{Conclusions}

Overall, our study finds that lncRNA-AK097647 expression is increased in both EV71-infected $\mathrm{RD}$ cells and patients. The enforced expression of IncRNA-AK097647 leads to a reduction in the mRNA level of IFN- $\lambda 1$, blocks
IFN $-\lambda 1$ secretion, and decreases the phosphorylation of $N F-\kappa B$ in human RD cells, thus resulting in a significant increase in the replication of EV71. Conversely, the knockdown of lncRNA-AK097647 upregulates IFN- $\lambda 1$ mRNA levels, increases IFN- $\lambda 1$ secretion, and induces the phosphorylation of NF- $\kappa \mathrm{B}$, thus resulting in a significant decrease in EV71 replication. These data suggest that lncRNA-AK097647 may be a potential therapeutic target for antiviral infection; however, this needs to be verified in future studies.

\section{Acknowledgements}

We thank the Medical Structural Biology Research Center of Wuhan University for technical assistance in confocal microscopy assay and RT-qPCR.

\section{Statement of Ethics}

The study was conducted according to the principles of the Declaration of Helsinki and approved by the Institutional Review Board of the College of Basic Medical Sciences, Wuhan University, in accordance with the guidelines for the protection of human subjects. Written informed consent was obtained from each participant.

\section{Conflict of Interest Statement}

The authors declare no conflicts of interest.

\section{Funding Sources}

This work was supported by the Fundamental Research Funds for Shenzhen Science and Technology Innovation Committee (No. JCYJ20170818143952175), the National Natural Sciences Foundation of China (No. 52073022, 81371790, 81641093, 81371422,81571481 and 31170154), Major AIDS and Viral Hepatitis and Other Major Infectious Disease Prevention and Control project of China (No. 2014ZX10001003), The Funds for Creative Research Groups of Hubei Natural Science Foundation (No. 2017CFA017), the Fundamental Research Funds for the Central Universities of China, and the Translational Medical Research Fund of Wuhan University School of Medicine.

\section{Author Contributions}

W.L. and M.C. conceived and designed the assays. B.Z., H.T., M.L., L.H., Y.H., L.L., S.H., J.Y., B.P., and X.H. analyzed the data. L. and M.C. wrote the manuscript. W.L., B.P., and X.H. revised the manuscript. All authors critically reviewed the manuscript and provided their final approval. 


\section{References}

1 Shih SR, Stollar V, Li ML. Host factors in enterovirus 71 replication. J Virol. 2011 Oct; 85(19):9658-66.

2 Huang KA, Zhou D, Fry EE, Kotecha A, Huang PN, Yang SL, et al. Structural and functional analysis of protective antibodies targeting the threefold plateau of enterovirus 71. Nat Commun. 2020 Oct 16;11(1):5253.

3 Ang LW, Phoon MC, Wu Y, Cutter J, James L, Chow VT. The changing seroepidemiology of enterovirus 71 infection among children and adolescents in Singapore. BMC Infect Dis. 2011 Oct 11:11:270.

4 Liu Y, Zhang F, Fu C, Wu S, Chen X, Shi Y, et al. Combination of intratypic and intertypic recombinant events in EV71: a novel evidence for the "triple-recombinant" strains of genotype A viruses in Mainland China from 2008 to 2010. Virus Genes. 2015 Jun;50(3):365-74.

5 Cawley S, Bekiranov S, Ng HH, Kapranov P, Sekinger EA, Kampa D, et al. Unbiased mapping of transcription factor binding sites along human chromosomes 21 and 22 points to widespread regulation of noncoding RNAs. Cell. 2004 Feb 20;116(4):499-509.

6 Guttman M, Rinn JL. Modular regulatory principles of large non-coding RNAs. Nature. 2012 Feb 15;482(7385):339-46.

7 Weidle UH, Birzele F, Kollmorgen G, Rüger $\mathrm{R}$. Long non-coding RNAs and their role in metastasis. Cancer Genomics Proteomics. 2017 May-Jun;14(3):143-60.

8 Zur Bruegge J, Einspanier R, Sharbati S. A long journey ahead: long non-coding RNAs in bacterial infections. Front Cell Infect Microbiol. 2017;7:95.

9 Xu S, Yang W, Yuan P, Yan J, Tang Y, Zheng $\mathrm{Y}$, et al. The long-noncoding RNA lnc-NONH enhances the early transcription of prototype foamy virus via upregulating expression of miR-34c-5p and Tas protein. Intervirology. 2019;62(3-4):156-63.

$10 \mathrm{Ma} \mathrm{H}$, Han P, Ye W, Chen H, Zheng X, Cheng $\mathrm{L}$, et al. The long noncoding RNA NEAT1 exerts antihantaviral effects by acting as positive feedback for RIG-I signaling. J Virol. 2017 May 1;91(9):e02250-16.

11 Wang $\mathrm{P}, \mathrm{Xu}$ J, Wang Y, Cao X. An interferonindependent lncRNA promotes viral replication by modulating cellular metabolism. Science. 2017 Nov 24;358(6366):1051-5.

12 Yin Z, Guan D, Fan Q, Su J, Zheng W, Ma W, et al. IncRNA expression signatures in response to enterovirus 71 infection. Biochem Biophys Res Commun. 2013 Jan 11;430(2): 629-33.
13 Meng J, Yao Z, He Y, Zhang R, Yang H, Yao $\mathrm{X}$, et al. Long non-coding RNA expression profiles in different severity EV71-infected hand foot and mouth disease patients. Biochem Biophys Res Commun. 2017 Dec 2; 493(4):1594-600.

14 Li Y, Zhang C, Qin L, Li D, Zhou G, Dang D, et al. Characterization of critical functions of long non-coding RNAs and mRNAs in rhabdomyosarcoma cells and mouse skeletal muscle infected by enterovirus 71 using RNA-Seq. Viruses. 2018 Oct 11;10(10):556.

15 Ouyang J, Zhu X, Chen Y, Wei H, Chen Q, Chi $X$, et al. NRAV, a long noncoding RNA, modulates antiviral responses through suppression of interferon-stimulated gene transcription. Cell Host Microbe. 2014 Nov 12; 16(5):616-26

16 Li J, Chen C, Ma X, Geng G, Liu B, Zhang Y, et al. Long noncoding RNA NRON contributes to HIV-1 latency by specifically inducing tat protein degradation. Nat Commun. 2016 Jun 13;7:11730.

17 Fujimoto Y, Kyogoku K, Takeda K, Ozaki K, Yamamoto S, Suyama H, et al. Antiviral effects against influenza A virus infection by a short hairpin RNA targeting the non-coding terminal region of the viral nucleoprotein gene. J Vet Med Sci. 2019 Mar 14;81(3):383-8.

18 Frantz R, Teubner L, Schultze T, La Pietra L, Müller C, Gwozdzinski K, et al. The secRNome of listeria monocytogenes harbors small noncoding RNAs that are potent inducers of beta interferon. mBio. 2019 Oct 8;10(5).

19 Fernando TR, Contreras JR, Zampini M, Rodriguez-Malave NI, Alberti MO, Anguiano J, et al. The lncRNA CASC15 regulates SOX4 expression in RUNX1-rearranged acute leukemia. Mol Cancer. 2017 Jul 19;16(1):126.

20 Li L, Chen H, Gao Y, Wang YW, Zhang GQ, $\mathrm{Pan} \mathrm{SH}$, et al. Long noncoding RNA MALAT1 promotes aggressive pancreatic cancer proliferation and metastasis via the stimulation of autophagy. Mol Cancer Ther. 2016 Sep;15(9): 2232-43.

21 Yu SY, Dong B, Zhou SH, Tang L. LncRNA MALAT1: a potential regulator of autophagy in myocardial ischemia-reperfusion injury. Int J Cardiol. 2017 Nov 15;247:25.

22 Huang J, Yang Y, Fang F, Liu K. MALAT1 modulates the autophagy of retinoblastoma cell through miR-124-mediated stx17 regulation. J Cell Biochem. 2018 May;119(5):385363.
23 Yuan P, Cao W, Zang Q, Li G, Guo X, Fan J. The HIF-2 $\alpha$-MALAT1-miR-216b axis regulates multi-drug resistance of hepatocellular carcinoma cells via modulating autophagy. Biochem Biophys Res Commun. 2016 Sep 23; 478(3):1067-73.

24 Garcia-Sastre A. Ten strategies of interferon evasion by viruses. Cell Host Microbe. 2017 Aug 9;22(2):176-84.

25 Yi C, Zhao Z, Wang S, Sun X, Zhang D, Sun $\mathrm{X}$, et al. Influenza A virus PA antagonizes interferon- $\beta$ by interacting with interferon regulatory factor 3. Front Immunol. 2017;8: 1051.

26 Gack MU, Diamond MS. Innate immune escape by dengue and West Nile viruses. Curr Opin Virol. 2016 Oct;20:119-28.

27 Werder RB, Lynch JP, Simpson JC, Zhang V, Hodge NH, Poh M, et al. PGD2/DP2 receptor activation promotes severe viral bronchiolitis by suppressing IFN- $\lambda$ production. Sci Transl Med. 2018 May 9;10(440):eaao0052.

28 Lundberg R, Melén K, Westenius V, Jiang M, Österlund $\mathrm{P}$, Khan $\mathrm{H}$, et al. Zika virus nonstructural protein NS5 inhibits the RIG-I pathway and interferon lambda 1 promoter activation by targeting IKK epsilon. Viruses. 2019 Nov 4;11(11): 1024

29 Cao T, Li X, Xu Y, Zhang S, Wang Z, Shan Y, et al. Npro of classical swine fever virus suppresses type III interferon production by inhibiting IRF1 expression and its nuclear translocation. Viruses. 2019 Oct 31;11(11): 998.

30 Wang C, Sun M, Yuan X, Ji L, Jin Y, Cardona CJ, et al. Enterovirus 71 suppresses interferon responses by blocking Janus kinase (JAK)/signal transducer and activator of transcription (STAT) signaling through inducing karyopherin-alpha1 degradation. J Biol Chem. 2017 Jun 16;292(24):10262-74.

31 Liu Y, Zhang Z, Zhao X, Yu R, Zhang X, Wu $S$, et al. Enterovirus 71 inhibits cellular type I interferon signaling by downregulating JAK1 protein expression. Viral Immunol. 2014 Aug;27(6):267-76.

32 Lu J, Yi L, Zhao J, Yu J, Chen Y, Lin MC, et al. Enterovirus 71 disrupts interferon signaling by reducing the level of interferon receptor 1 . J Virol. 2012 Apr;86(7):3767-76.

33 Li Y, Xie J, Xu X, Wang J, Ao F, Wan Y, et al. MicroRNA-548 down-regulates host antiviral response via direct targeting of IFN $-\lambda 1$. Protein Cell. 2013 Feb;4(2):130-41. 